\title{
Relevance, ad hoc concepts and analogy
}

\author{
Ewa Mioduszewska \\ University of Warsaw, Poland
}

\begin{abstract}
In Relevance Theory (RT) concepts are "enduring elementary mental structure[s] capable of playing different discriminatory or inferential roles on different occasions in an individual's mental life." (Sperber \& Wilson, 2012, p. 35). They may be lexicalized atomic concepts, ad hoc atomic concepts not encoded in our linguistic system and some innate concepts (Carston, 2010, p. 14). Concepts may be shared between interlocutors, idiosyncratic but grounded in common experience or fully idiosyncratic and non-communicable. They are "arrived at through the mutual pragmatic adjustment of explicature and contextual implicatures." (Carston, 2010, p. 10). Ad-hoc concepts are "pragmatically derived, generally ineffable, non-lexicalized [...] rough indication to aid readers in understanding what we have in mind in particular cases." (Carston 2010, p. 13). Concepts encoded will only occasionally be the same as the ones communicated because words are used to convey indefinitely many other ad hoc concepts constructed in a given context (Sperber \& Wilson, 2012, p. 43). Apparently, RT restricts the construction of ad hoc concepts by the search for relevance (definitions of (optimal) relevance, principles of relevance and relevance-theoretic comprehension procedure) and the potential connection (narrowing or broadening) between the denotations of the encoded and constructed concepts. The mechanisms underlying category narrowing/broadening seem not to be explicitly described and explained. What provides a very general but, at the same time, precise account of concept-relatedness is Hofstadter \& Sander's (2013) understanding of analogy. The question posed here is whether this understanding may help explain concept-relatedness in Relevance Theory.
\end{abstract}

Keywords: Relevance Theory, analogy, ad hoc concepts, concept-relatedness

In Relevance Theory, in both its standard version (Sperber \& Wilson, 1986/95) and later extensions (Carston, 2012; 2013), no unique cognitive mechanism of ad hoc concept construction is identified. Rather, such concepts emerge as a result of category narrowing/broadening in the process of "the mutual pragmatic adjustment of explicature and contextual implications" (Carston, 2010, p. 10), triggered by communicators' search for relevance in the standard version whereas their construction from non-conceptual word meaning is left unaccounted for (Mioduszewska, 2015).

Hofstadter and Sander's (2013) Analogy Theory (AT) claims analogy to be the cognitive process underlying conceptualization and categorization. The ongoing, constant, automatic, spontaneous analogy-making process explains and predicts construction or retrieval of ad hoc 
concepts. The theory does not explain, however, what stops the process in individual cases of utterance comprehension. Relevance Theory predicts that processing stops when communicators' expectations of relevance are satisfied.

\section{Relevance Theory (RT)}

Relevance Theory (Sperber \& Wilson, 1986/95), a theory of communication and cognition, is a model of ostensive-inferential (verbal) communication the fundamental assumptions of which are the two principles of relevance, the concept of (optimal) relevance and relevance-theoretic comprehension procedure.

Two principles of relevance:

\section{Cognitive Principle of Relevance}

Human cognition tends to be geared to the maximization of relevance (Wilson \& Sperber, 2004, p. 7).

\section{Communicative Principle of Relevance}

Every ostensive stimulus conveys a presumption of its own optimal relevance (Wilson \& Sperber, 2004, p. 9).

The definitions of relevance:

\section{Relevance of an input to an individual}

a. Other things being equal, the greater the positive cognitive effects activated by processing the input, the greater the relevance of the input to the individual at that time.

b. Other things being equal, the greater the processing effort expended, the lower the relevance of the input to the individual at that time (Wilson \& Sperber, 2004, p. 10).

\section{Optimal relevance}

"An ostensive stimulus is optimally relevant to an audience iff: (a) It is relevant enough to be worth the audience's processing effort; (b) It is the most relevant one compatible with communicator's abilities and preferences" (Wilson \& Sperber, 2004, p. 10).

When searching for the relevance of an ostensive stimulus, the hearer/reader uses the relevancetheoretic comprehension procedure, which reads as follows:

Relevance-theoretic comprehension procedure:

a. Follow a path of least effort in computing cognitive effects. Test interpretive hypotheses [...] in order of accessibility;

b. Stop when your expectations of relevance are satisfied (Wilson \& Sperber, 2004, p. 13).

Sub-tasks in the overall comprehension procedure

a. Constructing an appropriate hypothesis about explicit content (in relevance-theoretic terms, explicatures) via decoding, disambiguation, reference resolution, and other pragmatic enrichment processes.

b. Constructing an appropriate hypothesis about the intended contextual assumptions (in relevance-theoretic terms, implicated premises).

c. Constructing an appropriate hypothesis about the intended contextual implications (in relevance-theoretic terms, implicated conclusions) (Wilson \& Sperber, 2004, p. 16). 
The fundamental assumptions constitute tools of describing and explaining the comprehension process during any form of communication triggered by recognizing an ostensive stimulus, which comes with a guarantee of its own optimal relevance (communicative principle of relevance). Since the stimulus is assumed to be relevant, the communicator processes it in order to maximize its relevance, as predicted by the cognitive principle of relevance. Processing the stimulus follows the relevance-theoretic comprehension procedure. In constructing hypotheses about the speaker's explicit and implicit meaning, the hearer recognizes, activates and/or constructs concepts for which the stimulus provides clues.

Relevance Theory predicts the (mental) existence of a variety of concepts such as lexicalized atomic concepts, mental concepts not encoded in our linguistic system, some innate concepts (Carston, 2010, p. 14) and newly constructed (or possibly retrieved) ad hoc concepts.

Concepts are "enduring, elementary mental structure[s] capable of playing different discriminatory or inferential roles on different occasions in an individual's mental life" (Sperber \& Wilson, 2012, p. 35). Ad hoc concepts are "pragmatically derived, generally ineffable, nonlexicalized [...] rough indication to aid readers in understanding what we have in mind in particular cases" (Carston, 2010, p. 13). Ephemeral representations of particulars and complex conceptual structures are not concepts, while stabilized, idiosyncratic, non-lexicalized concepts based on private experience are concepts (Sperber \& Wilson, 2012, pp. 35-36). Lexicalized (encoded) atomic concepts and ad hoc concepts are related.

\section{Concept-relatedness in Relevance Theory}

In Relevance Theory,

[...], the words in a language can be used to convey not only the concepts they encode, but also indefinitely many other related concepts to which they might point in a given context. [...] The occurrence of a word in an utterance provides a piece of evidence, a pointer to a concept involved in the speaker's meaning. [...] It may so happen that the intended concept is the very one encoded by the word, which is therefore used in its strictly literal sense (Sperber \& Wilson, 2012, p. 43).

In every case, the conveyed concept has to be contextually worked out. Here, Example I illustrates the process.

Example 1 (Wilson, 2011, pp. 198, 207)

Context: Two friends John and Peter discuss their friend Robert.

John: How good a friend is Robert?

Peter: Robert is a computer

sentence uttered: Robert is a computer

lexical meaning of computer: COMPUTER ${ }^{1}$ (robert) (a type of machine)

\footnotetext{
Capital letters mark concepts.
} 
On this interpretation, Peter does not find the utterance to be relevant enough, so he must have meant COMPUTER ${ }^{\star}$ (robert) (a type of machine + some humans). John does not find the first interpretation COMPUTER (robert) to be relevant enough, so he continues processing, arriving at COMPUTER ${ }^{\star \star}$ (robert) (a type of machine + some humans + ?) with the implicature Robert lacks feelings, processes information well, etc. The contextually worked out COMPUTER $^{\star *}$ is related to the encoded concept COMPUTER. Such concept relatedness rests on the relatedness of the concepts' denotations and the potential relatedness of the concepts' logical entries. The final interpretation of the constructed concepts COMPUTER ${ }^{\star}$ and COMPUTER $^{\star *}$ is conditioned by the search for relevance, following the two principles of relevance.

The content or semantics of a concept is its denotation. The encoded concept's (COMPUTER) denotation (a type of machine) is its meaning. The word encoding the concept (computer) inherits its denotational semantics (a type of machine).

The concepts communicated by the use of a word (computer) (COMPUTER*, COMPUTER $^{\star *}$ ) have a narrower (more specific) or/and broader denotation (a type of machine + some humans + ?) than the encoded concept (COMPUTER). The relatedness of denotations reflects concepts-relatedness (computer-like human and non-human entities, including computers).

Another way of accounting for concept-relatedness comes from the assumption that the content or semantics of a concept is a set of inference rules (logical entry), constituting the truth-conditional semantic content of a concept. The relatedness of the logical entries of COMPUTER, COMPUTER ${ }^{\star}$ and COMPUTER ${ }^{\star *}$ should account for the relatedness among the three concepts. This works on the assumption that words map onto addresses in memory: lexical, encyclopaedic and logical entries of concepts, with encyclopaedic entries providing grounds for drawing implicated premises in utterance interpretation.

The third relevance-theoretic mechanism accounting for concept-relatedness is the communicators' search for relevance of the utterance interpretation. In Example I, Peter's search for relevance of Robert is a computer leads him to COMPUTER ${ }^{\star}$, John's search for relevance of the same utterance possibly leads him to COMPUTER ${ }^{\star *}$, in both cases resulting in the relatedness of COMPUTER, COMPUTER ${ }^{\star}$ and COMPUTER ${ }^{\star *}$.

To sum up, concept-relatedness in Relevance Theory is encapsulated by three mechanisms.

(1) Concept-relatedness results from their denotational relatedness (narrowing and/or broadening a concept's denotation).

(2) Concept-relatedness results from the similarity and/or difference of the concepts' logical entries.

(3) Concepts-relatedness is an outcome of the hearer's search for the relevance of the interpretation of the speaker/writer's ostensive stimulus, as predicated by the two principles of relevance, the understanding of relevance itself and the relevance-theoretic comprehension procedure.

The first two mechanisms are valid on the assumption that a concept's denotation and logical entries are always determinable. The examples below cast doubts on the possibility of these requirements being fulfilled. 
Example II (Hofstadter \& Sander, 2013, pp. 228-229)
a) This animal is a pig.
[PIG] (animal)
b) This girl is a pig.
$\left[\mathrm{PIG}^{*}\right]$ (dirty and sloppy entity)
c) This damn word processor is a pig.
$\left[\mathrm{PIG}^{\star *}\right]$ (negative sloppy entity)

The denotational relatedness of the concepts PIG, PIG*, $\mathrm{PIG}^{\star \star}$ is not easily (if at all) determinable. Neither is the logical entry of $\mathrm{PIG}^{\star \star}$ available.

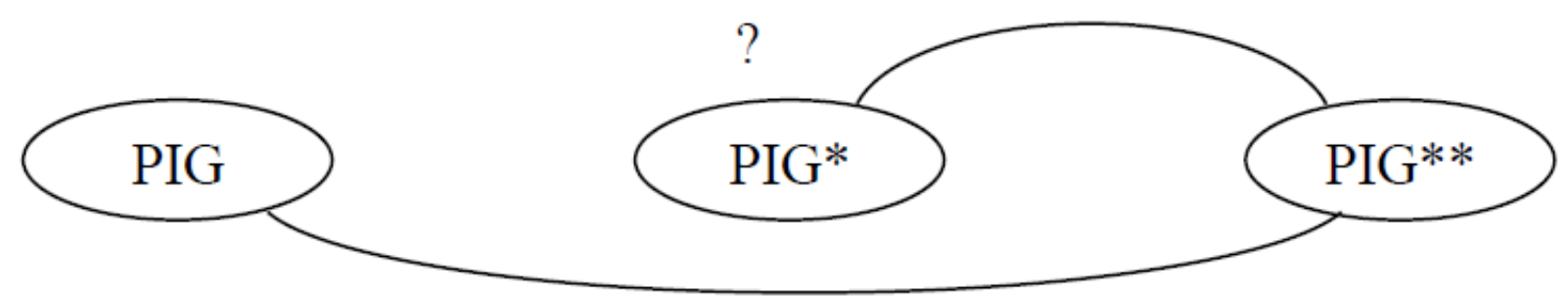

$?$

Figure 1: Concept-relatedness in RT (a). The denotational and inferential relatedness of the three concepts is not determinable.

The only basis for the construction of $\mathrm{PIG}^{\star}, \mathrm{PIG}^{\star \star}$ ad hoc concepts is the communicators' search for relevance.

Example III (Hofstadter \& Sander, 2013, pp. 185-186)
a) Let's have coffee.
[COFFEE] (a typical coffee)
b) Coffee?
$\left[\mathrm{COFFEE}^{\star}\right]$ (hot drink after a meal)
c) Let's go and have some coffee.
$\left[\mathrm{COFFEE}^{\star *}\right.$ ] (a quick (social) beverage)

In this example, the denotational and inferential relatedness of the three concepts COFFEE, $\mathrm{COFFEE}^{\star}$ and $\mathrm{COFFEE}^{\star *}$ is not easily determinable. Again, the only ground for the concepts' construction and relatedness is the communicators' search for relevance.

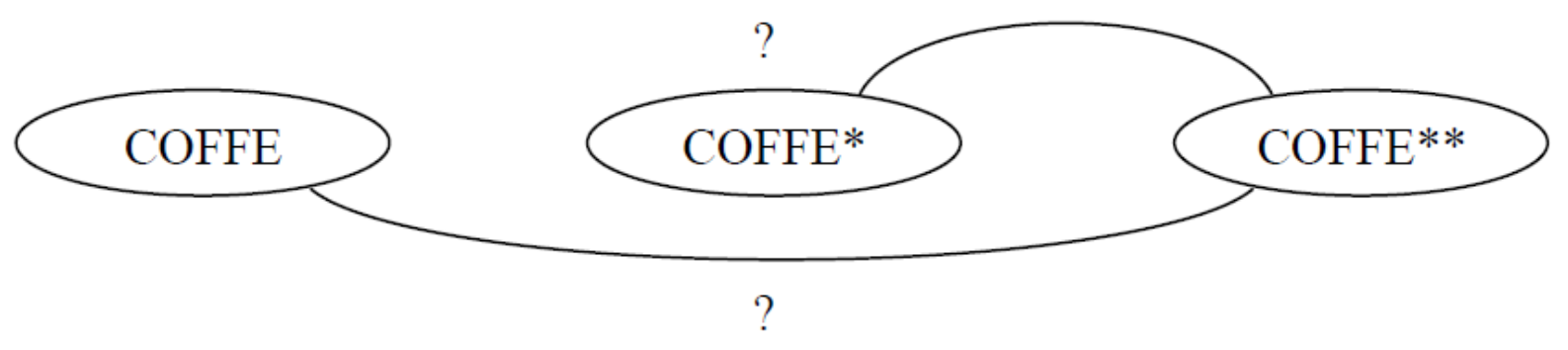

Figure 2: Concept-relatedness in $R T(b)$.

Hofstadter and Sander's (2013) Analogy Theory provides a mechanism of conceptrelatedness that sheds new light on ad hoc concept construction. 


\section{Analogy Theory (AT)}

According to Hofstadter and Sander (2013), analogy-making is the essence of thinking and cognition. Analogy-making, categorization and conceptualization are in fact three labels describing the same phenomenon. Since there is no thought without concepts and there are no concepts without analogy, analogies are "the fire and fuel of thinking" (Hofstadter \& Sander, 2013).

Analogy is the mapping of some mental structure on another mental structure with there being some similarity/resemblance between the structures, via abstraction, that is analogydriven category extension (e.g. generalizing abstraction B ----- A, with B being a subcategory of A), resulting in markedness of narrower categories and the unmarkedness of broader categories.

Analogy is the very essence of thought, being a fundamental, automatic and widespread cognitive process, occurring below the conscious threshold. The triggering of memories by analogy is the essence of being human. Analogy works at all levels of verbal and non-verbal communication. Humans compare what is happening to them now with what happened to them in the past. For example, the proverb One should never try to catch a falling knife activates an analogy-making driven concept of huge, irrepressible forces against which one has no power and which would carry one off to one's doom if one were to risk to try to stop them (Hofstadter \& Sander, 2013, p. 28).

Analogy maps one mental structure on another mental structure. New concepts (e.g. HUB) influence 'more primitive' concepts (AIRPORT). At first there was "airport", and then "hub". But once hub exists, airport is influenced. In the same vein, the act of metaphorization is a way of extending our categories, the goal being to understand more directly and intensely that which surrounds us.

The mental structures on which analogy operates are conceptual in nature. In AT concepts equal categories. They are mental structures created over time, which evolve and contain information in an organized way, allowing access to it.

A concept/category is "an abstract pattern in the brain that stands for some regular, recurrent aspect of the world [...] to which any number of different words/'signs' point." (Hofstadter \& Sander, 2013, p. 34). Concepts may be labelled or unlabelled, as in the case of the concept which may be verbalized as "that time I found myself locked outside my house in bitterly freezing weather because the door slammed shut by accident." (Hofstadter \& Sander, 2013, p. 20). Conceptualization is automatic and biologically bestowed. For example, the stimuli one perceives being at an airport allow for the recovery or construction of categories via an automatic, unconscious process of triggering them, and this comes about the way of analogy with familiar categories (Hofstadter \& Sander, 2013, p. 33).

Such categorization/conceptualization is the graded, grey, shaded linking of an entity (situation) to a prior category in one's mind, giving the feeling of understanding and anticipating future events. The same non-stop categorization through analogy-making applies from the mundane to the sophisticated understanding of utterances.

Concepts, that is mental structures, consist of a specific/concrete instance and a halo. Concepts' halos account for the vague blurring and flexible quality of the concept's core. It thins 
out as one moves further out from the core. The fuzzy boundaries of categories are inherent in the act of categorization (Hofstadter \& Sander, 2013, p. 62).

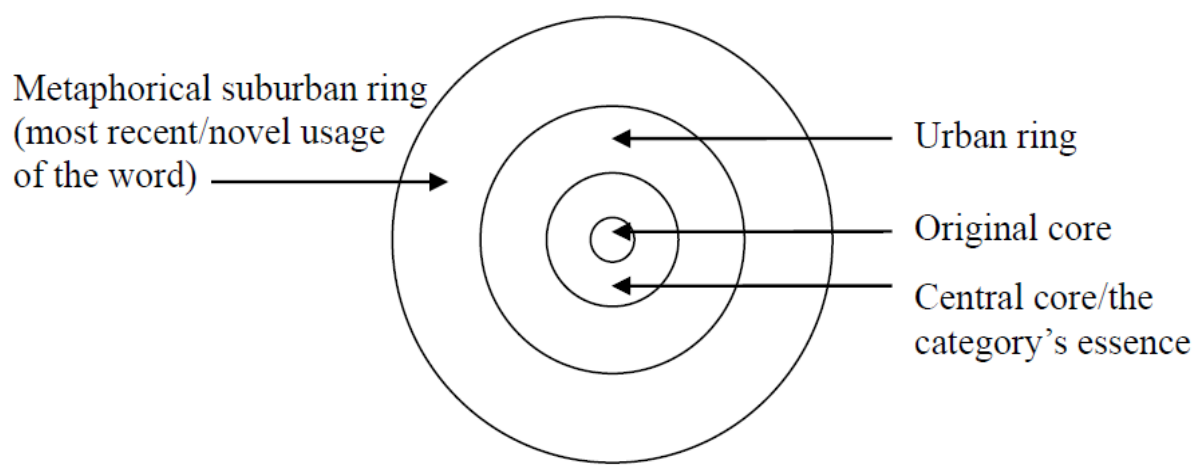

Figure 3: Concept scheme in AT.

The scheme is dynamic. Mental categories have tiny, almost solid central cores, which can move over time whereas outer rings and shells reflect repeated acts of extension due to perceived analogies. In this way what was once metaphorical gradually may become the essence.

\begin{abstract}
A category has an ancient core, some commercial zones, some residing zones, an outer ring, and then suburbs that $[\ldots]$ shade off into countryside. One passes smoothly and continuously from a concept's core to its fringes. It results from a spectrum of analogies from the simplest to the most far-fetched. (Hofstadter \& Sander, 2013, p. 65).
\end{abstract}

Concepts, which equal categories, are the outcomes of a long series of spontaneous analogies between a freshly perceived stimulus and the old mental analogy with only one member or a highly developed mental category based on the same mechanism. Their core meaning is their denotation (abstract skeleton). Their halos are their periphery. Concepts, both their cores and peripheries, exist in multidimensional spaces.

\footnotetext{
The idea of a multidimensional space in which concepts exist, somewhat like separate points (core), however around each such point there is a halo that accounts for the vague blurry and flexible quality of the concept and this halo becomes even more tenuous as one moves further out from the core. (Hofstadter \& Sander, 2013, p. 50).
}

Concepts are densely stitched together through relations of similarity and context. In multidimensional conceptual spaces, the most common concepts for a culture, era, etc. constitute the core items (for example body parts, classes of animals, plants, things to eat and drink). Near the centre, there are concepts which (quasi) universally convey part of the human conditions. Rings and shells of conceptual spaces are their periphery. Rings are less frequently used concepts (for example thanks, barn, fog) and shells are not frequent at all (for example frowning, fingernail-biting, income tax). The process of categorization/ conceptualization is ongoing because "the human mind is [...] seeking novelty" (Hofstadter \& Sander, 2013, p. 65) in order to understand that which surrounds us. As a consequence, the set of metaphors at one's disposal cannot be limited or fixed. 
The process of encoding is conceptual because purely image-based processes would be insufficient. Events are encoded (perceived, distilled, stored) in terms of the prior concepts that we have acquired.

All language expressions: words, phrases, idioms, proverbs, formulaic expressions, fables, grammatical patterns are conceptual in nature. All words involve concepts and are conceptrelated. Categories/concepts, which are mental entities, are more numerous than words, which is explained by the existence of words with multiple senses, categories with no verbal labels, categories expressed by phrases, compound words, acronyms, idiomatic phrases and idioms, sentences and sentence fragments, proverbs and fables.

\section{Concept-relatedness in Analogy Theory}

In Analogy Theory, concepts extend through spontaneous analogies, growing in generality or/and becoming more discriminatory. Further distinctions, required and achieved by experts, co-occur with establishing broader categories. Both processes are visible in compounds, idioms, proverbs, catch phrases as well as in building up concepts with no verbal labels.

A single item (entity) belongs to lots of categories. Our mental life consists in placing entities in one category and then in reassigning them to another category. Context changes categorization and modifies our perception. The members of a category change with the given context. A single word in a given language can denote different categories, and at different levels of category abstractions. "In language [...] category membership [is] shifting [...] thanks to the phenomenon of marking, which allows an entity to shift its category membership without changing its lexical label, but simply by changing the level of abstraction that applies to that level” (Hofstadter \& Sander, 2013, p. 193).

Objects and situations move from one category to another easily and unconsciously. Such flexibility and creativity result from the human faculty of extending categories and making maps between them. Concepts develop through category extensions from one member, through cores and halos. Analogy is the motor of all such extensions. The extension is the result of the fact that the human mind has the abilities to abstract to deal with the world's vast diversity.

Examples II and III (recalled below) illustrate different levels of abstraction.

Example II

Lexical label: pig

a) PIG: concrete category, animal

b) PIG $^{*}$ category of higher level of abstraction, dirty and sloppy entity

c) $\mathrm{PIG}^{* *}$ : utmost level of abstraction, negative, sloppy entity

The three concepts: PIG, $\mathrm{PIG}^{\star}, \mathrm{PIG}^{\star \star}$ are related by the abstraction level (from core to periphery). 


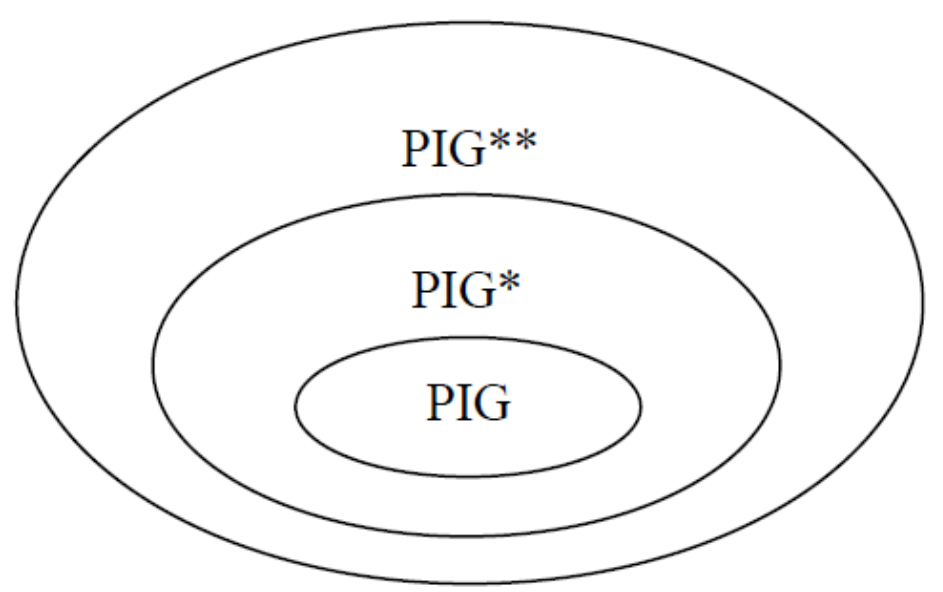

Figure 4: Concept-relatedness in AT (a).

Example III

Lexical label: coffee

a) COFFEE: smallest abstraction level, a typical coffee

b) COFFEE*: one abstraction level up, an after-meal hot drink

c) COFFEE $^{* *}$ utmost level of abstraction, a quick (social) beverage

The three concepts: COFFEE, COFFEE ${ }^{*}$ COFFEE $^{* *}$ are related by the abstraction level (from core to periphery).

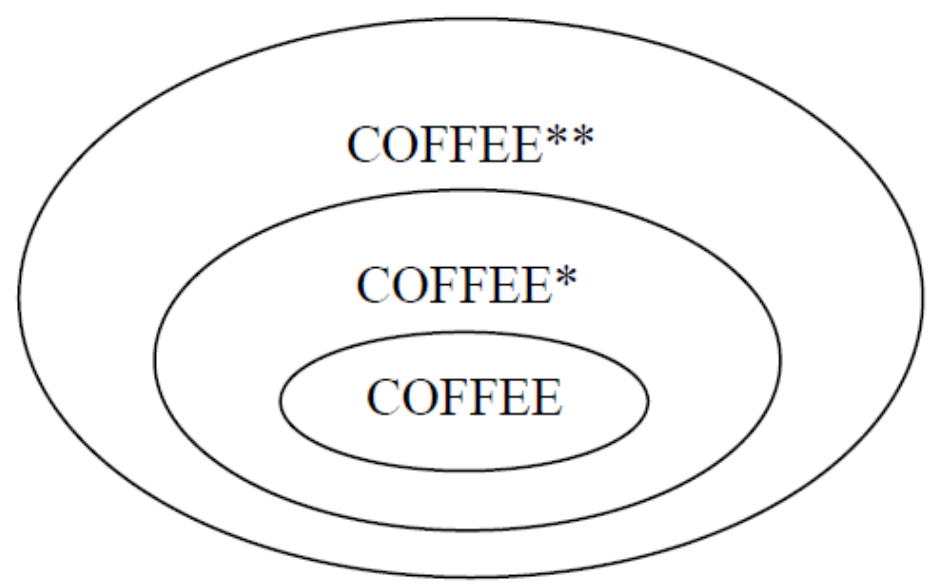

Figure 5: Concept-relatedness in AT (b)

The words pig and coffee, depending on context, may lead to different conceptualizations by spontaneous analogy-making between the new stimulus and old concepts in memory. The parallel inference-making in each case is understood as introducing new mental elements into a situation that one is facing. Such elements find room in the concepts' halos. Any situation can be categorized in a limitless number of different fashions. Sometimes we wish to make distinctions, and sometimes we wish to see commonalities. Distinguishing things consists in aligning them to different categories. Our adaptability results from our ability to categorize things in different ways. That is why using the same words by different people may mean 
different categories. That is why 'metaphorical' meanings may take on a life of their own, becoming autonomous new meanings.

In analogy-based approach, in which conceptualization and categorization are near synonyms, category/concept extensions/abstractions explain how and why one label may activate a variety of concepts and show how they are related.

The question that does arise in this approach is what makes the analogy-making process stop. Here Relevance Theory provides an answer - communicators stop processing when their expectations of relevance are satisfied, as predicted by the relevance-theoretic comprehension procedure.

\section{Relevance, analogy and ad hoc concepts}

Both Relevance Theory and Analogy Theory are general, powerful cognitive theories. Among their goals, explaining the comprehension process is one that they share. Both the theories base on some general cognitive principles: for Relevance Theory they are the cognitive and communicative principles of relevance, whereas for Analogy Theory it is the automatic, spontaneous analogy-making process. Apart from the principles, both theories assume the existence of a psychologically real mechanism of stimuli interpretation. In Relevance Theory, it is the relevance-theoretic comprehension procedure, and in Analogy Theory, it is the process of category extension through the process of abstraction. Both theories propose an interpretation of categorization and conceptualization, claiming at the same time its psychological reality, generality or even universality.

In the two theories, concepts are defined in a similar vein. In Relevance Theory, concepts are "enduring elementary mental structure[s] capable of playing different discriminatory or inferential roles on different occasions in an individual's mental life." (Sperber \& Wilson, 2012, p. 35). In Analogy Theory, a concept/category is " an abstract pattern in the brain that stands for some regular, recurrent aspect of the world [...] to which any number of different words/'signs' point" (Hofstadter \& Sander, 2013, p. 34).

Both theories assume that the human mental lexicon is vaster than the set of lexicalized concepts. According to Relevance Theory, "the words can be used to convey not only the concepts they encode but also indefinitely many other related concepts to which they may point in a given context" (Sperber \& Wilson, 2012, p. 43). According to Analogy Theory, a single word in a given language can denote different entities and a single item (entity) may belong to lots of categories (Hofstadter \& Sander, 2013, p. 193).

Both theories agree that the content (meaning) of a concept is its denotation.

Although similar in some general goals, Relevance Theory and Analogy Theory also differ in many important aspects. Relevance Theory is a theory not only of cognition but also of communication, so one of its main goals is to explain how people communicate. The proposed account is a model of ostensive-inferential communication, stemming from Paul Grice's theory of inferential communication, but significantly extended and elaborated in relevance-theoretic terms. Analogy Theory claims that analogy-making is not only responsible for categorization or conceptualization processes but also constitutes the very essence of thinking. 
The two theories also differ in what types of concepts they admit. In Relevance Theory, concepts are divided into lexicalized atomic concepts, ad hoc concepts and other concepts not encoded in our linguistic system, possibly also some innate concepts. In the standard version of the theory, lexicalized concepts are accessed through three addresses in memory: lexical, logical and encyclopaedic to which the relevant word takes the communicators. In Analogy Theory, only one type of concepts is assumed. All concepts have cores and peripheries (halos), all of them are both input to and output of the analogy-making process. What is more, according to Analogy Theory, all language expressions are conceptual in nature: words, phrases, sentences, sentence fragments, longer texts such as fables, idioms, proverbs, metaphorical expressions, aphorisms, syntactic and potentially even phonological structures. In Relevance Theory, not all language expressions are claimed to be conceptual in nature. The theory introduces the idea of procedural meaning, the existence of pro-concepts or even of non-conceptual, non-semantic schematic word meaning (Carston, 2012; 2013).

As discussed at length in "Some remarks on non-conceptual word meaning and truthconditional content in Robyn Carston's pragmatics” (Mioduszewska, 2015):

\begin{abstract}
The main theses of Robyn Carston's $(2013 ; 2012)$ pragmatics, concerning the stable/standing meaning of three open-class items, that is nouns, verbs and adjectives are as follows: (1) Stable (lexicalized) word meanings are non-conceptual, that is non-semantic (Carston, 2013, p. 184). Words do not encode full-fledged concepts; in fact, there is no literal, encoded meaning of lexical items (Carston, 2013, p. 200). As in RT lexical pragmatics, words are merely pointers to or evidence for a speaker's meaning; (2) "Each word comes with its own distinct but schematic meaning, which functions as a constraint on the general pragmatic process of accessing or constructing a concept, a process which is wholly motivated by the goal of the pragmatic system which is to deliver speaker meaning." (Carston, 2013, p. 200). (3) In view of the schematic, non-conceptual stable meaning of open-class words, the pragmatic inferential process of constructing (contextual) ad-hoc concepts becomes obligatory (Carston, 2013, p. 187). This process allows for only one of a range of concepts to be the first one accessed or constructed, as determined by considerations of relevance; (4) Word meanings are conceptually underspecified (Carston, 2013, pp. 184, 187, 196, 201). (Mioduszewska, 2015, pp. 6-7)
\end{abstract}

This development of Relevance Theory does not provide any mechanism of concept construction that would allow for the question of concept-relatedness to be answered.

Relevance Theory and Analogy Theory also differ in their interpretation of ad hoc concepts and their construction. Hofstadter \& Sander (2013) do not introduce the notion of ad hoc concepts at all because there is no need for them in their theory. Ad hoc concepts in Relevance Theory are 'regular' concepts in AT by means of category extensions and abstractions.

As already stated, in Relevance Theory, ad hoc concepts are "pragmatically derived generally ineffable, non-lexicalized [...] rough indication to aid readers in understanding what we have in mind in particular cases." (Carston, 2010, p. 13). They are arrived at through the mutual pragmatic adjustment of explicatures and contextual implications. Any activation of the process of category narrowing and broadening is explained by the search for relevance, but the nature of the process is not fully explained. 
Another important difference between the two theories is their understanding of inference. In Analogy Theory, inference is understood as introducing any new mental element in the comprehension process. In Relevance Theory, with its modular view of the mind, the inferential deductive (logical) device is a separate mental module which allows the theory to postulate the existence of elimination deductive rules and, derivatively, of analytic, synthetic and contextual implications and implicatures.

In Analogy Theory, the analogy-making process maps a mental structure on another mental structure, with some similarity or resemblance between the structures secured by the fact that the structures are linked via category abstraction, that is analogy-driven category extension, resulting in markedness of narrower categories and unmarkedness of broader categories. In Relevance Theory, the connection between the (possibly encoded) atomic concepts and ad hoc concepts derives from the relatedness of the concepts' denotations and their logical entries. The content of the relatedness depends on the fulfilment of communicators' search for relevance, thus being individual-relative.

In Analogy Theory, concepts exist in a multidimensional space, like separate points (cores) around which there is a halo accounting for the flexible quality of the concept. In Relevance Theory, concepts do not have cores and peripheries, but the utterances of which they are part may trigger strong, core-like implicatures and an array of weak implicatures, which perform the role of a concept's periphery.

Thus, the word computer in Example I in Relevance Theory activates the lexicalized atomic concept COMPUTER (machine). Since this concept does not secure the utterance's relevance, in the process of mutual pragmatic adjustment, new ad hoc concepts COMPUTER ${ }^{\star}$, COMPUTER $^{\star \star}$ are created. The three concepts are distinct.

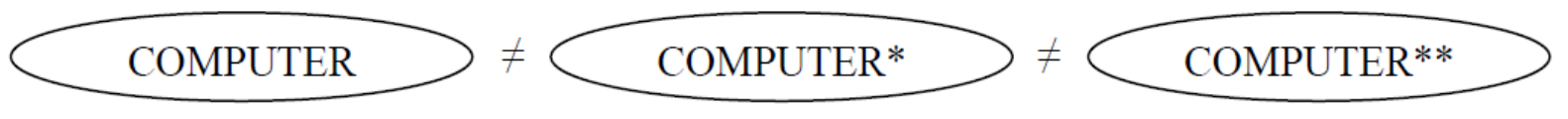

Figure 6. Distinct concepts in RT

The relatedness of these three concepts exists if the search for relevance requires the same, wherein it should derive from the relatedness of the three concepts' denotations and of their logical entries. However, the denotations of COMPUTER ${ }^{\star}$ and COMPUTER ${ }^{\star *}$ are not determinable, so the relatedness among them cannot be established. In Analogy Theory, the lexicalized concept COMPUTER constitutes the core of the concept the periphery of which, that is COMPUTER ${ }^{\star}$ and COMPUTER ${ }^{\star *}$, is related to the core by way of category abstraction. 


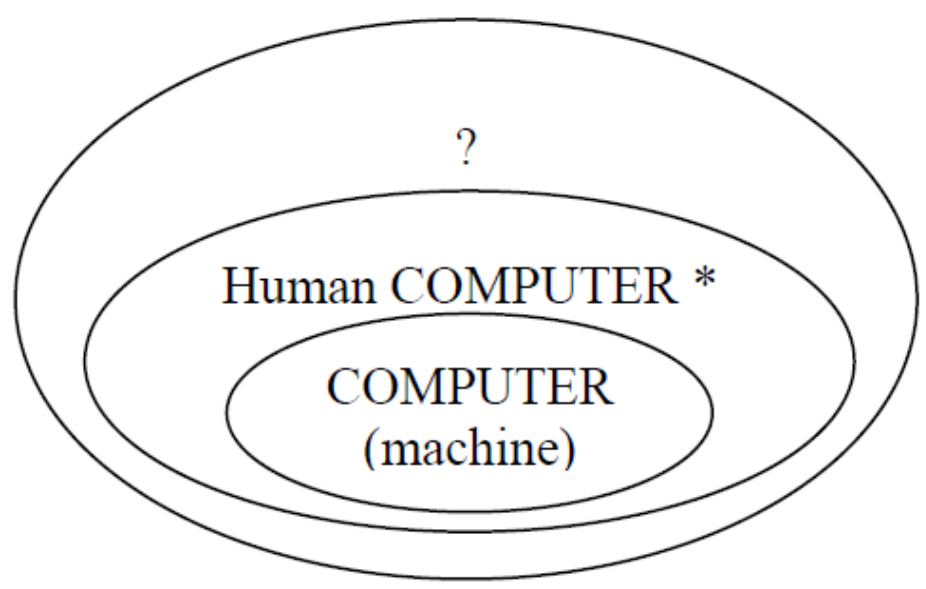

Figure 7: Related concepts in AT

Here, the concept-relatedness is predicted by the very nature of the theory itself and follows from it automatically. As it has already been mentioned, "in language category membership [is] shifting thanks to the phenomenon of marking, which allows an entity to shift its category membership without changing its lexical label, but simply by changing the level of abstraction [...]" (Hofstadter \& Sander, 2013, p. 93).

To sum up, in establishing concept-relatedness between lexicalized and ad hoc concepts, Relevance Theory faces problems if denotational relatedness is not determinable or if logical entries are not available. No such problems arise in Analogy Theory, when it comes to establishing concept relatedness. And so AT helps explain concept-relatedness in RT. On the other hand, RT provides constraints on the analogy-making process.

\section{Conclusions}

The aim of what has been presented here has been to justify two claims. The first one is that the analogy-theoretic process of analogy-making, if incorporated into Relevance Theory, would help explain concept-relatedness in RT. The second claim is that relevance-theoretic comprehension procedure, if incorporated into Analogy Theory, would help explain what stops the analogy-making process in individual cases of utterance (stimuli) interpretation.

However, to accept these claims as valid, it would be necessary to examine the potential (in)compatibility of the two theories at the level of very fundamental assumptions and to analyze carefully the results of such incorporations for the theories. Until this is done, the two claims made above should rather be treated as, hopefully, interesting and intriguing possibilities.

\section{References}

Carston, R. (2010). Lexical pragmatics, ad hoc concepts and metaphor: A Relevance Theory perspective. Italian Journal of Linguistics, 22(1), 157-180.

Carston, R. (2012). Word meaning and concept expressed. The Linguistic Review, 29, 607-623. 
Carston, R. (2013). Word Meaning, What is Said and Explicature. In C. Penco \& F. Domaneschi (Eds.), What is Said and What is Not (175-203). Stanford, California: CSLI publications.

Hofstadter, D. \& Sander, E. (2013). Surfaces and Essences. Analogy as the Fuel and Fire of Thinking. New York: Basic Books.

Mioduszewska, E. (2015). Some remarks on non-conceptual word meaning and truth-conditional content in Robyn Carston's pragmatics. Acta Philologica, 46, 5-17.

Sperber, D. \& Wilson, D. (1986[1995]). Relevance. Communication and cognition. Oxford: Blackwell.

Sperber, D. \& Wilson, D. (2012). The mapping between the mental and the public lexicon. In. D. Wilson \& D. Sperber (Eds.), Meaning and Relevance (pp. 31-47). Cambridge: CUP.

Wilson, D. (2011). The Conceptual-Procedural Distinction: Past, Present and Future. In V. Escandell-Vidal, M. Leonetti \& A. Ahern (Eds.), Procedural Meaning: Problems and Perspectives (pp. 3-31). UK: Emerald Group Publishing.

Wilson, D. \& Sperber, D. (2004). Relevance theory. In L. Horn \& G. Ward (Eds.), Handbook of pragmatics (pp. 607-632). Oxford: Blackwell. 